\title{
Tuning the mechanosensitivity of a BK channel by changing the linker length
}

\author{
Hucheng Zhao ${ }^{1,2}$, Masahiro Sokabe $e^{2,3,4}$ \\ ${ }^{1}$ Institute of Biomechanics and Medical Engineering, Tsinghua University, Beijing 100084, PR China; ${ }^{2}$ Department of Physiology, \\ Nagoya University Graduate School of Medicine School, 65 Tsurumai, Nagoya 466-8550, Japan; ${ }^{3}$ International Cooperative Research \\ Project / Solution Oriented Research for Science and Technology, Cell Mechanosensing Project, Japan Science and Technology \\ Agency, Nagoya 466-8550, Japan; ${ }^{4}$ Department of Molecular Physiology, National Institute for Physiological Sciences, Okazaki \\ 444-8585, Japan
}

Some large-conductance $\mathrm{Ca}^{2+}$ and voltage-activated $\mathrm{K}^{+}(\mathrm{BK})$ channels are activated by membrane stretch. However, the mechanism of mechano-gating of the BK channels is still not well understood. Previous studies have led to the proposal that the linker-gating ring complex functions as a passive spring, transducing the force generated by intracellular $\mathrm{Ca}^{2+}$ to the gate to open the channel. This raises the question as to whether membrane stretch is also transmitted to the gate of mechanosensitive (MS) BK channels via the linker-gating complex. To study this, we changed the linker length in the stretch-activated BK channel (SAKCaC), and examined the effect of membrane stretch on the gating of the resultant mutant channels. Shortening the linker increased, whereas extending the linker reduced, the channel mechanosensitivity both in the presence and in the absence of intracellular $\mathrm{Ca}^{2+}$. However, the voltage and $\mathrm{Ca}^{2+}$ sensitivities were not significantly altered by membrane stretch. Furthermore, the SAKCaC became less sensitive to membrane stretch at relatively high intracellular $\mathrm{Ca}^{2+}$ concentrations or membrane depolarization. These observations suggest that once the channel is in the open-state conformation, tension on the spring is partially released and membrane stretch is less effective. Our results are consistent with the idea that membrane stretch is transferred to the gate via the linker-gating ring complex of the MS BK channels.

Keywords: BK channel, mechanosensitive channel, mechano-gating, heart

Cell Research (2008) 18:871-878. doi: 10.1038/cr.2008.88; published online 29 July 2008

\section{Introduction}

Mechanosensitive (MS) ion channels are found in a variety of organisms from bacteria to human. These channels transduce mechanical stimuli into intracellular signals, such as elevation in intracellular $\mathrm{Ca}^{2+}$ concentration and electrical activities [1-6], which are important in many physiological functions and pathological conditions, for example, sound detection [4], cell volume regulation [7], touch sensation [8], hypo-osmotic shock protection [9], cell locomotion [10], muscular dystrophy and cardiac arrhythmias [11]. The BK channel is one type of MS channels which is widely expressed in both excitable and non-excitable cells and plays an important role in, for example,

Correspondence: Hucheng Zhao ${ }^{\mathrm{a}}$, Masahiro Sokabe ${ }^{\mathrm{b}}$

E-mail: ${ }^{a} z h a o h c @ m a i l . t s i n g h u a . e d u . c n ;{ }^{b}$ msokabe@med.nagoya-u.ac.jp Received 14 October 2007; revised 3 January 2008; accepted 18 February 2008; published online 29 July 2008 vascular smooth muscle tone regulation, neuronal firing and endocrine cell secretion [12-16]. The MS properties of the BK channels expressed in skeletal muscle [17], kidney [18], smooth muscle [19], neuroepithelial cells and osteoblasts $[20,21]$ have been characterized. We previously identified a stretch-activated BK channel (referred as $\mathrm{SAKCaC}$ ) in cultured chick embryo ventricular myocytes [22]. Like other BK channels, SAKCaC can be activated by membrane voltage and intracellular $\mathrm{Ca}^{2+}$. However, the unique property of this channel is that it can also be activated by membrane stretch. Molecular cloning and subsequent functional characterization of the SAKCaC have shown that the channel subunit contains a 59-aminoacid insert (called stress-axis regulated exon (STREX)) in the C-terminus, and that STREX domain is the stretch sensor conferring the membrane stretch sensitivity to the channel [23, 24]. However, it is not well understood how the membrane stretch is transferred to the gate to open the channel. In the model based on the MthK channel structure, 
the pore-forming a subunit contains two regulator of $\mathrm{K}^{+}$ conductance (RCK) domains in the intracellular C-terminus, and eight RCK domains from the tetrameric channel form a gating ring $[25,26]$. The linker that connects the S6 gate to the RCK domain and the gating ring form the linker-gating complex, which functions as a passive spring and transduces the force generated by intracellular $\mathrm{Ca}^{2+}$ to the channel gate [27]. The STREX domain is located between RCK1 and RCK2 domains in the long intracellular C-terminus of SAKCaC (Figure 1) [19]. The present study showed that shortening the linker rendered the channel more sensitive to membrane stretch, whereas lengthening the linker resulted in the opposite effect, suggesting that, similar to $\mathrm{Ca}^{2+}$ activation, the membrane stretch sensed by the STREX domain seems to be transmitted to the channel gate by the linker-gating complex.

\section{Results}

\section{Activation of SAKCaC by membrane stretch}

We first characterized the activation of wild-type (WT) $\mathrm{SAKCaC}$ by membrane stretch in excised inside-out patches. Figure 2A shows typical single channel current traces in response to stepwise negative pressures with 10 $\mathrm{mmHg}$ increments. Figure 2B plots the channel open probability $\left(P_{\mathrm{o}}\right)$ against the level of pressure applied. The $P_{\mathrm{o}}$ increased with increased pressure. In contrast, there were no detectable channel activities induced by membrane stretch in mock transfected cells (data not shown). The $\mathrm{SAKCaC}$ activation occurred in the absence of intracellular $\mathrm{Ca}^{2+}$, suggesting that membrane stretch directly activated the channel. All these results are in agreement with those reported previously $[9,23,28]$.

\section{Effect of linker length on $P_{\circ}$ of SAKCaC}

To address the role of the linker in SAKCaC activation, we produced mutant channels, in which the S6-RCK1 linker was shortened by deleting one residue or lengthened by inserting three residues. Figure $3 \mathrm{~A}$ shows representative current traces of the mutant BK channels in response

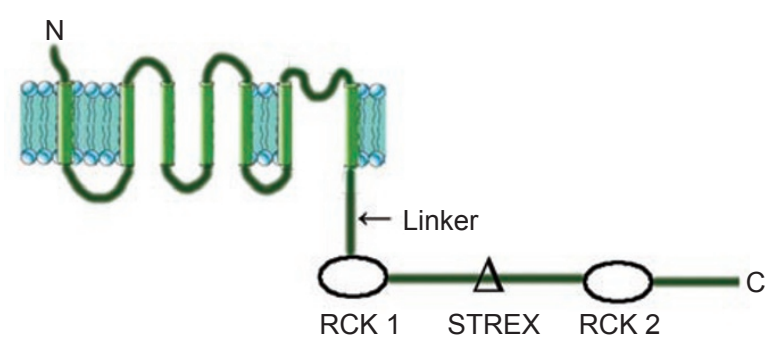

Figure 1 Schematic presentation of the linker, RCK1, RCK2 and STREX domains of BK channel $\alpha$-subunit.
A
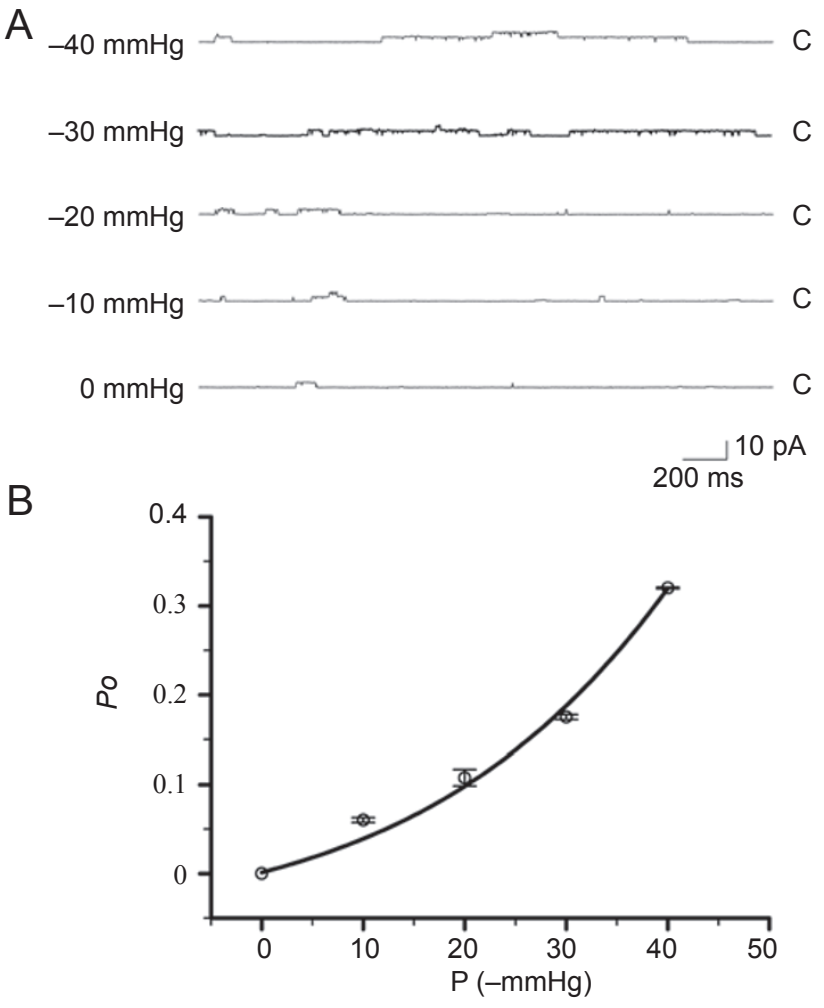

Figure 2 Pressure dependency of $P_{\circ}$ of SAKCaC. Pipette solution contained $145 \mathrm{mM} \mathrm{K}^{+}, 0 \mathrm{mM} \mathrm{Ca}^{2+}$ (with $1 \mathrm{mM} \mathrm{EGTA}$ ), bath solution contained $145 \mathrm{mM} \mathrm{K}^{+}$and $0 \mathrm{mM} \mathrm{Ca}^{2+}(n=18)$. (A) Representative single-channel current traces of SAKCaC in response to membrane stretch ( $\mathrm{C}$ denotes the closed current level). Membrane potential was held at $+60 \mathrm{mV}$. (B) Pressure- $P_{0}$ curve was fit by the Boltzmann equation.

to membrane depolarization $(+60 \mathrm{mV})$, and Figure $3 \mathrm{~B}$ summarizes the $P_{\mathrm{o}}$. It is obvious that the $P_{\mathrm{o}}$ was greatly increased by shortening the linker $(-1)$, but decreased by lengthening the linker $(+3)$. Similar results were reported previously in the Slo BK channels and the interpretation is that the linker-gating ring complex forms a passive spring to exert the force on the gate [27]. We also generated mutant subunits in which more than one residue was deleted or more than three residues were inserted in the linker. However, no detectable channel activities were observed in cells expressing these mutants, even at voltages of greater than $+100 \mathrm{mV}$ and in the presence of $100 \mu \mathrm{M}$ intracellular $\mathrm{Ca}^{2+}$ (data not shown), possibly due to defective channel assembly and/or membrane trafficking.

Effect of changing the linker length on the mechanosensitivity of $\mathrm{SAKCaC}$

We next examined the effect of changing the linker length on SAKCaC activation by membrane stretch. As shown in Figure 4, membrane stretch greatly increased the 
$P_{\mathrm{o}}$ of both WT and mutant channels in a dose-dependent manner. However, the mechanosensitivity was strongly enhanced by shortening the linker $(-1)$, and decreased by increasing the linker $(+3)$. The relationship of $P_{\mathrm{o}}$ to the pressure, resulting from membrane stretch, could be fitted with the Boltzmann equation: $P_{\mathrm{o}}=1 /\left[1+\exp \left(p-p_{1 / 2}\right) k\right]$, where $p$ is the pressure, $p_{1 / 2}$ is the pressure required to induce half-maximal channel activation, and $k$ is the reciprocal of the curve steepness or slope. The $p_{1 / 2}$ values were $23 \mathrm{~mm}$ $\mathrm{Hg}, 18 \mathrm{~mm} \mathrm{Hg}^{*}$, and $27 \mathrm{~mm} \mathrm{Hg}^{*}$, and the $k$ values were $22 \mathrm{~mm} \mathrm{Hg}, 23 \mathrm{~mm} \mathrm{Hg}$, and $20 \mathrm{~mm} \mathrm{Hg}$ for WT, -1, and +3 mutant channels, respectively. The $p_{1 / 2}$ values for both mutant channels were significantly different from that for WT $(*$ indicate significant difference $p \& 1 t ; 0.05)$. The unitary channel conductance $(260 \pm 3.1 \mathrm{pS})$, however, remained unaltered by either membrane stretch or change in the linker length (data not shown).

Effect of changing the linker length on the mechanosensitivity of SAKCaC at different membrane voltages and intracellular $\mathrm{Ca}^{2+}$ concentrations

One key feature of the BK channels is $\mathrm{Ca}^{2+}$ and voltage dependence of channel activation [12]. Here we therefore examined the role of the linker in $\mathrm{Ca}^{2+}$-dependent $\mathrm{SAKCaC}$

A

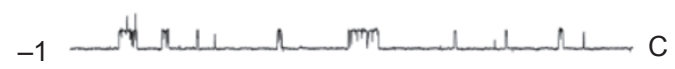

WT

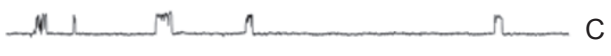

$+3$
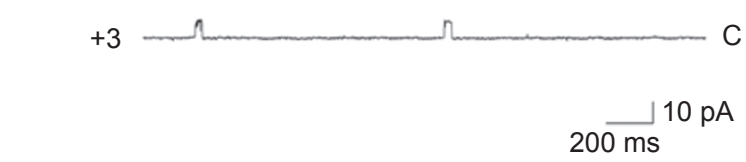

B

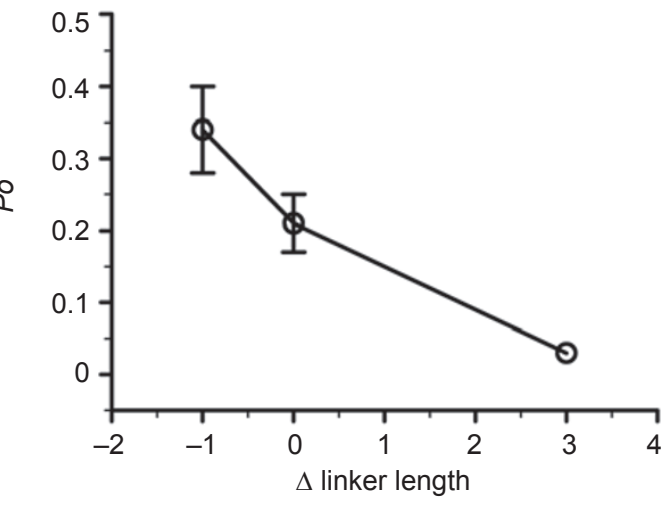

Figure 3 Change in the linker length alters the channel activity in the absence of $\mathrm{Ca}^{2+}(n=11)$. (A) Representative recording of currents at a membrane voltage of $+60 \mathrm{mV}$. (B) Plot of $P_{\mathrm{o}}$ versus change in the linker length.

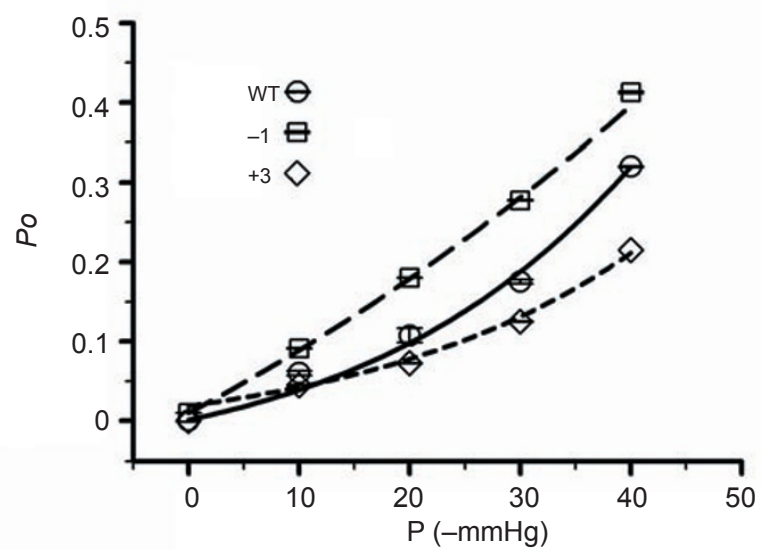

Figure 4 Mechanosensitivity of SAKCaC with linkers of different length $(n=10)$. The pipette and bath solution contained $145 \mathrm{mM}$ $\mathrm{K}^{+}$with $0 \mathrm{mM} \mathrm{Ca}^{2+}$. Membrane potential was held at $+60 \mathrm{mV}$. The data points were fitted with the Boltzmann equation.

activation in the presence of membrane stretch. As shown in Figure 5, increase in intracellular $\mathrm{Ca}^{2+}$ concentration from 0 to $1.2 \mu \mathrm{M}$ greatly enhanced the channel activity of both WT and mutant channels at all the levels of membrane stretch tested. Compared to WT, the $P_{\mathrm{o}}-\mathrm{Ca}^{2+}$ curve for the mutant channel with a shorter linker $(-1)$ was left-shifted, and the slope was not significantly changed (Figure 5B). In contrast, the $P_{\mathrm{o}}-\mathrm{Ca}^{2+}$ curve was shifted to the right for the mutant channel with a lengthened linker $(+3)$, and the slope was also decreased (Figure 5C). In the presence of

Table 1 The effect of membrane stretch on the Hill coefficient of $\mathrm{SAKCaC}$ with a linker of different length

\begin{tabular}{cccc}
\hline $\begin{array}{c}\text { Negative pressure } \\
(\mathrm{mm} \mathrm{Hg})\end{array}$ & Wild & -1 & +3 \\
\hline-0 & 1.42 & 1.56 & 1.01 \\
-10 & 1.47 & 1.71 & 1.05 \\
-20 & 1.62 & 1.90 & 1.05 \\
-30 & 1.63 & 1.90 & 1.09 \\
-40 & 1.67 & 2.13 & 1.11 \\
\hline
\end{tabular}

Table 2 The effect of membrane stretch on V1/2 (mV) of SAKCaC with a linker of different length

\begin{tabular}{cccc}
\hline $\begin{array}{c}\text { Negative pressure } \\
(\mathrm{mm} \mathrm{Hg})\end{array}$ & $\begin{array}{c}\text { Wild } \\
\left(\mathrm{V}_{1 / 2}\right)\end{array}$ & $\begin{array}{c}-1 \\
\left(\mathrm{~V}_{1 / 2}\right)\end{array}$ & $\begin{array}{c}+3 \\
\left(\mathrm{~V}_{1 / 2}\right)\end{array}$ \\
\hline-0 & 92 & 55 & 110 \\
-10 & 82 & 47 & 88 \\
-20 & 50 & 28 & 82 \\
-30 & 12 & -25 & 50 \\
-40 & 0 & -27 & 46 \\
\hline
\end{tabular}




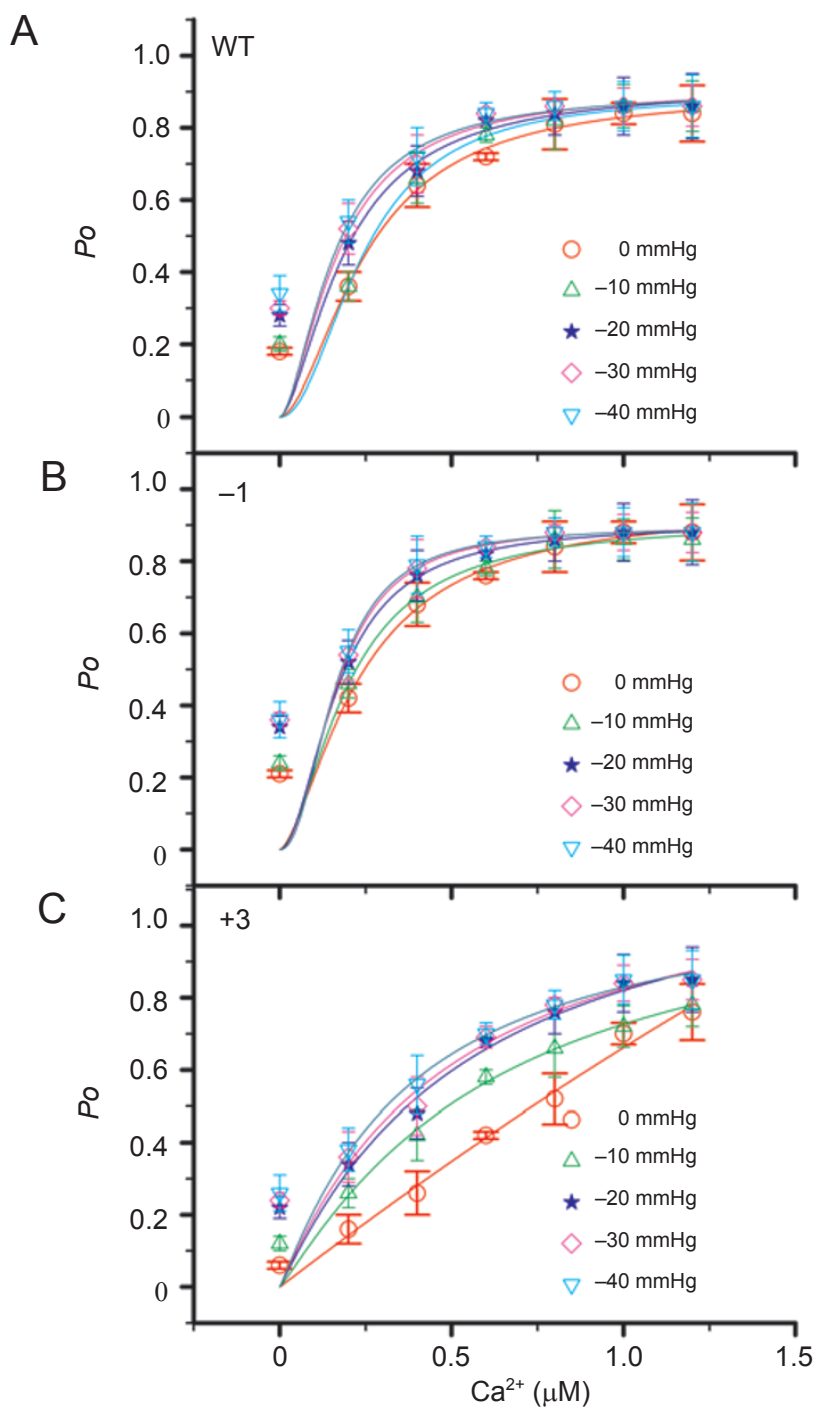

Figure 5 Effect of changing the linker length on $\mathrm{Ca}^{2+}$ dependence of SAKCaC in the presence of membrane stretch $(n=11)$. Membrane potential was held at $+60 \mathrm{mV}$. The data were fitted with the Hill equation: $P_{o}=\left[\mathrm{Ca}^{2+}\right]_{i}^{\eta H} /\left(K_{d}^{\eta H}+\left[\mathrm{Ca}^{2+}\right]_{i}^{\eta \dagger}\right)$, where $\eta \mathrm{H}$ is the Hill coefficient and $K_{d}$ is the dissociation constant.

intracellular $\mathrm{Ca}^{2+}$ concentrations, application of negative pressure induced an increase in the $P_{\mathrm{o}}$. Furthermore, for both WT and mutant channels, increase in negative pressure resulted in a parallel left-shift in the $P_{\mathrm{o}}-\mathrm{Ca}^{2+}$ curves without obvious change in the slope (Figure 5; Table 1). Thus, the enhanced SAKCaC activation in the presence of membrane stretch seems not due to an increase in $\mathrm{Ca}^{2+}$ sensitivity, suggesting that change in membrane stretch does not alter the $\mathrm{Ca}^{2+}$ sensitivity.

We also examined whether change in the linker length and membrane stretch affects the sensitivity of SAKCaC to membrane voltage. Figure 6 plots the $P_{\mathrm{o}}$ against the membrane voltage $(V)$ for the WT and mutant channels in the absence of intracellular $\mathrm{Ca}^{2+}$ and in the presence of membrane stretch ranging from 0 to $40 \mathrm{mmHg}$. These data were fitted with the Boltzmann equation to derive the voltage for half-channel activation, $V_{0.5}$. The results show that lengthening the linker increased, whereas shortening the linker decreased, the $V_{0.5}$ values (Table 2). In addition, compared to WT at all the membrane stretches tested, the $P_{\mathrm{o}}-V$ curve was left-shifted for the mutant channel with a shortened linker (Figure 6B), but right-shifted with decreased slopes for the mutant channel with a lengthened linker (Figure 6C). The $P_{\mathrm{o}}$ at fixed pressure was voltage-dependent, being higher when the membrane was depolarized.

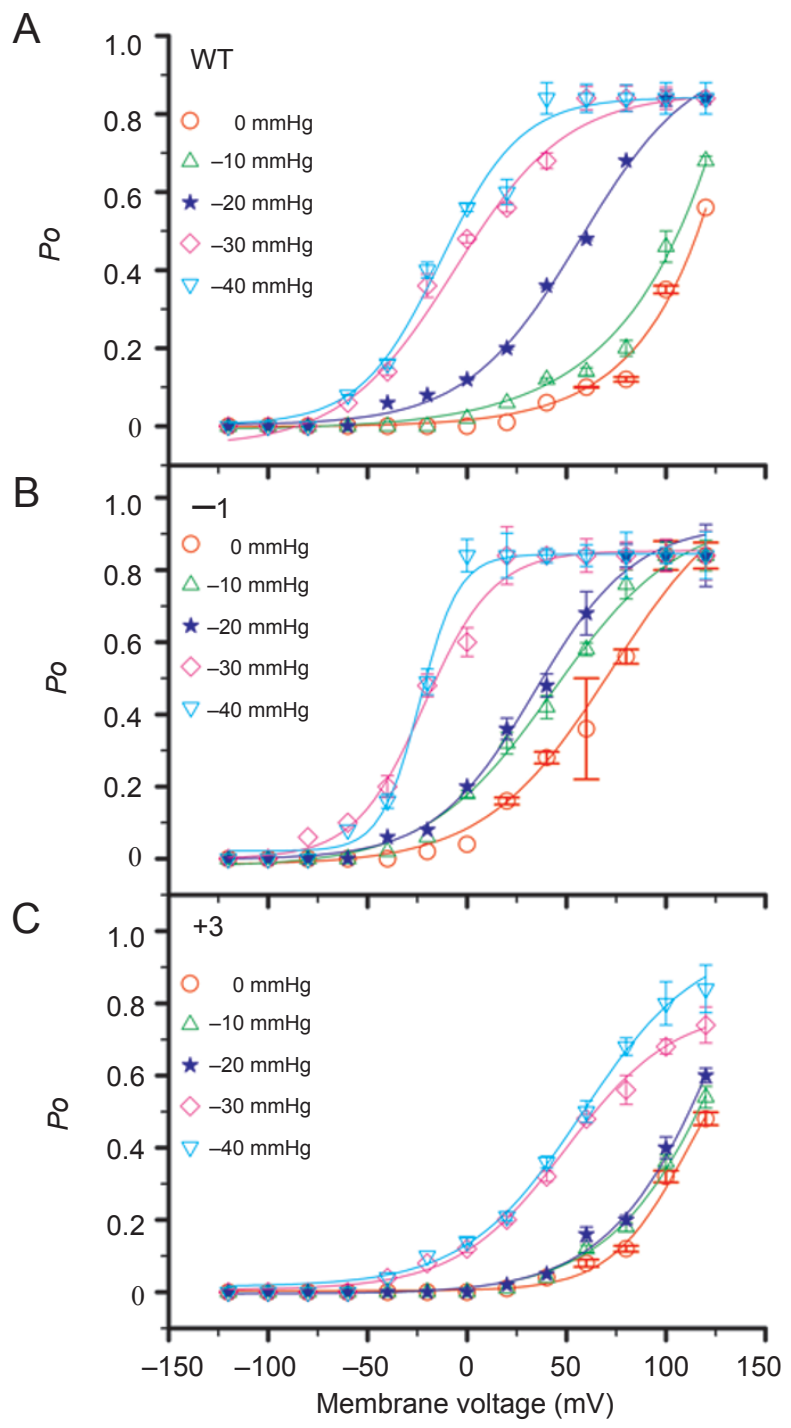

Figure 6 Effect of changing the linker length on voltage dependence of SAKCaC in the presence of membrane stretch in the absence of $\mathrm{Ca}^{2+}(n=16)$. The data were fitted with the Boltzmann equation: $P_{0}=1 /\left\{1+\exp \left[-Z F / R T\left(V-V_{1 / 2}\right)\right]\right\}$, where $V_{1 / 2}$ is the membrane potential for half-maximal channel activation. 


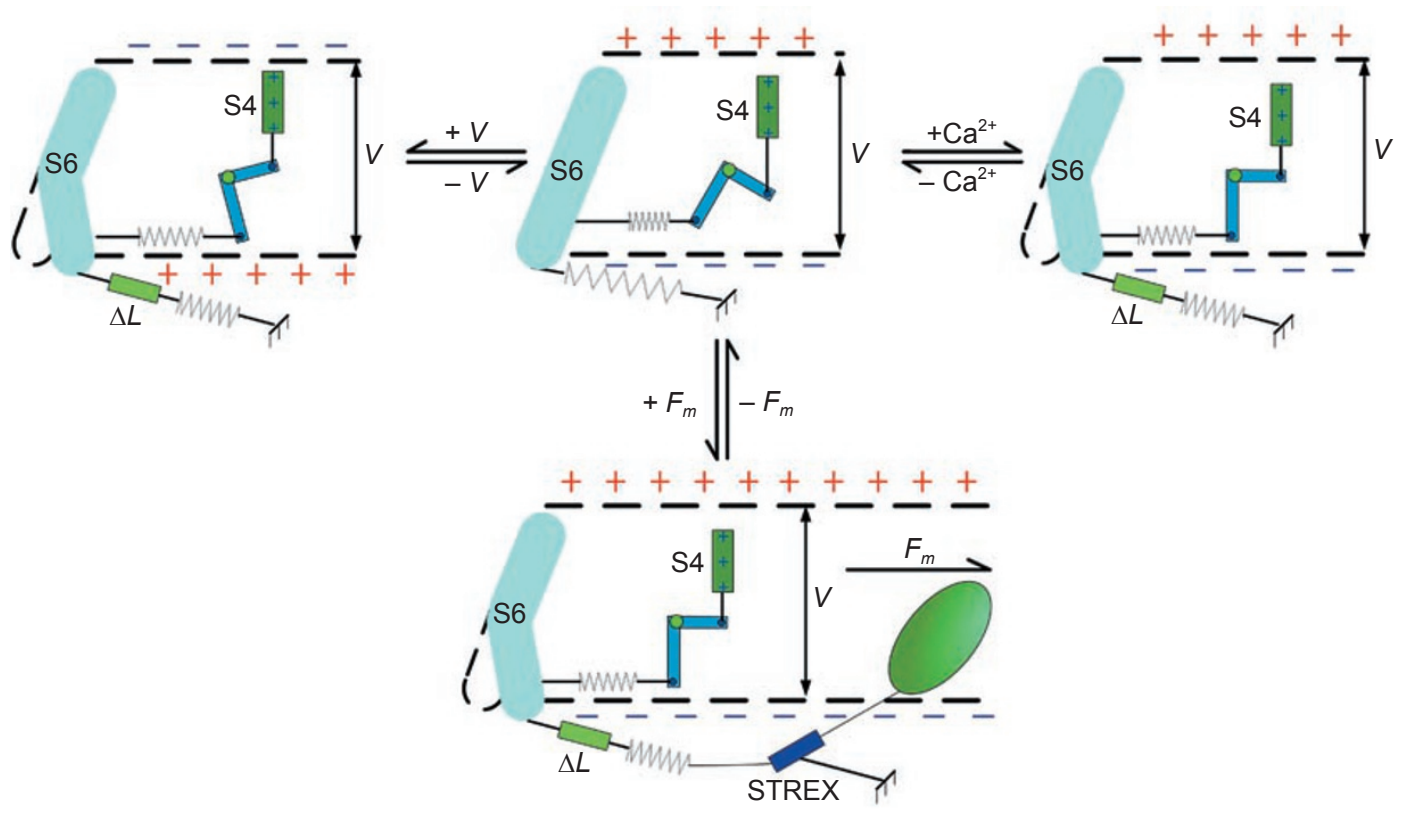

Figure 7 Regulation of BK channel gating by membrane stretch based on the spring model proposed for BK channel gating by Niu et al [27]. The membrane stretch (Fm) was conveyed by a membrane-associated protein and STREX, and then transferred to the gate by the linker connecting the transmembrane segment 6 (S6) gate. The charged transmembrane segment 4 (S4) voltage sensor is indirectly connected to the $\mathbf{S} 6$ gate through a spring. Addition of amino acids into the linker leads to changes of linker length $(\Delta L)$.

When the pressure was increased, the $P_{\mathrm{o}}-V$ curves were left-shifted. However, for both WT and mutant channels, there was almost no further increase in the slope at the relatively high membrane stretch. Our results also indicate that the channels were less sensitive to membrane stretch at relatively high intracellular $\mathrm{Ca}^{2+}$ concentrations (Figure 5) or membrane depolarization (Figure 6) (for original data see Supplementary information, Data S1).

\section{Discussion}

This study investigated the role of the linker in activation and particularly mechanosensitivity of SAKCaC. Membrane stretch induced an increase in the channel activity in a dose-dependent manner. The sensitivity to membrane stretch increased greatly by shortening the linker, and reduced by lengthening the linker, both in the presence and in the absence of intracellular $\mathrm{Ca}^{2+}$ (Figures 4 and 5). Furthermore, the $\mathrm{SAKCaC}$ was less sensitive to membrane stretch in the presence of relatively high intracellular $\mathrm{Ca}^{2+}$ concentrations or membrane depolarization. These results provide evidence consistent with the mechanical model recently proposed for the BK channel gating by Niu et al. [27]. In this model, eight RCK domains form the gating ring, and the linker that connects the S6 gate with RCK1 domain acts as a passive spring, transducing the force to the gate and pulling it open when the membrane is stretched. This model is able to account for the mechanosensitivity of SAKCaC. The membrane tension stretches the channel protein, in turn pulling the linker and increasing the tension on the spring. The tension on the spring opens the gate and thereby increases the SAKCaC activity. Thus we suggest that the membrane stretch is also transferred to the gate by the linker ring complex. More recently, we have demonstrated that the sensitivity of SAKCaC to membrane stretch is detected by the STREX domain locating between RCK1 and RCK2 in the intracellular C-terminus. Deletion of STREX or substitution of serine (S24) within the STREX domain diminishes the stretch sensitivity [28]. Thus, it is obvious that the mechanical forces are detected by the STREX domain and then transferred to the gate by the linker connecting the S6 gate with RCK1. Then a question arises as to how the STREX senses and transmits the force in the membrane to the channel. A simple explanation may be that the STREX domain interacts with a membrane-associated protein, which senses or transmits the force in the membrane, as illustrated in Figure 7. One notable feature of the SAKCaC channels with linkers of different lengths is the high $\mathrm{Ca}^{2+}$ sensitivity (Figure 5). The present study suggests that it may arise from the STREX domain, as previously described for the BK channels in human glioma cells [29]. It has been proposed that binding of $\mathrm{Ca}^{2+}$ to the 
gating ring may increase the $P_{\mathrm{o}}$ by expanding the gating ring to pull the channel open $[26,27,30]$. Therefore, we conclude that the force generated by $\mathrm{Ca}^{2+}$ or membrane stretch is transferred to the gate via a common pathway.

The influence of the linker length on the $\mathrm{Ca}^{2+}$ and voltage dependence of SAKCaC activation in the presence of membrane stretch was also examined. The $P_{\mathrm{o}}$ versus membrane potential $(V)$ or $\mathrm{Ca}^{2+}$ curves were shifted by altering the linker length; shortening the linker had little effect on the slopes of $\mathrm{Ca}^{2+}-P_{\mathrm{o}}$ curve and $V-P_{\mathrm{o}}$ curve, suggesting that shortening linker length does not significantly affect the voltage sensitivity and $\mathrm{Ca}^{2+}$ sensitivity of SAKCaC. However, lengthening the linker decreased the slopes of $P_{\mathrm{o}}-\mathrm{Ca}^{2+}$ curve and voltage- $P_{\mathrm{o}}$ curve, revealing that increasing the linker length decreases the effectiveness of $\mathrm{Ca}^{2+}$ and voltage activation of the channel. Furthermore, the $P_{\mathrm{o}}$ against membrane potential $(V)$ curve was left-shifted for $\mathrm{SAKCaC}$ when membrane stretch was applied. However, the change in membrane stretch from -10 to $-40 \mathrm{~mm} \mathrm{Hg}$ had little effect on the slope over the range of $P_{\mathrm{o}}$ values, suggesting that membrane stretch does not significantly affect the voltage sensitivity of SAKCaC. These results are consistent with the gating properties observed from the BK channels in mouse skeletal muscle fibers [30, 31], which were activated by membrane stretch without altering the voltage sensitivity. We also evaluated whether the $\mathrm{SAKCaC}$ activation by membrane stretch was due to an increase in $\mathrm{Ca}^{2+}$ sensitivity. We examined the effects of $\mathrm{Ca}^{2+}$ on the SAKCaC gating when membrane stretch was applied. The channel activity was enhanced by membrane stretch, resulting in a parallel left-shift of the $\mathrm{Ca}^{2+}-P_{\mathrm{o}}$ curve without affecting the slope, suggesting that $\mathrm{Ca}^{2+}$ sensitivity is not altered by membrane stretch. This is in agreement with the results previously described for the BK channels in smooth muscle cells [19] and skeletal muscle cells [32], whose activation in response to membrane stretch is not due to enhancement of $\mathrm{Ca}^{2+}$ sensitivity. Although activation of $\mathrm{SAKCaC}$ by membrane stretch is independent of the $\mathrm{Ca}^{2+}$ or voltage sensitivity, the spring-based gating model suggests that the effect of membrane stretch on the SAKCaC gating is similar to that produced by increasing intracellular $\mathrm{Ca}^{2+}$ or membrane depolarization, as illustrated in Figure 7. Binding of $\mathrm{Ca}^{2+}$ expands the gating ring and pulls the gate open, while depolarization opens the $\mathrm{SAKCaC}$ via the $\mathrm{S} 4$ voltage sensor pulling a spring to open the gate $[27,33]$. It should be emphasized that tension on the spring is essential for influences of $\mathrm{Ca}^{2+}$, membrane stretch, and voltage on the SAKCaC activation. The tension, caused by either membrane stretch or $\mathrm{Ca}^{2+}$ binding or membrane depolarization, would destabilize the closed conformation and favor the open conformation. The spring model predicts that the major effect of change in $\mathrm{Ca}^{2+}$ concentration or membrane potential occurs effectively only in the closed-state conformation (Figure 7). The effectiveness of SAKCaC activation by membrane stretch was reduced at relatively high intracellular $\mathrm{Ca}^{2+}$ or membrane depolarization, suggesting that when the channel is in open-state conformation, the membrane stretch also becomes less effective.

MS channels are found in many mammalian cell types, in which they are thought to be involved in a number of physiological or pathological functions. In the heart cells, the SAKCaC is thought to be involved in dilation or relaxation of heart. However, it still remains to be determined how $\mathrm{SAKCaC}$ functions in the heart under physiological and pathological conditions. The present study has provided evidence shedding light on how the channel is activated in response to membrane stretch.

\section{Materials and Methods}

\section{Mutagenesis and expression of $\mathrm{SAKCaC}$}

The a subunit of the MS BK channel or SAKCaC (accession number AB 072618) was previously cloned from a chick embryonic heart cDNA library [23]. Introduction of mutations in the linker between S6 and RCK1 domain was described previously [27]. In brief, the linker was shortened by deletion of one amino acid residue from GGSYSAVSG to GGSYAVSG (referred as -1), or lengthened by insertion of three amino acid residues from GGSYSAVSG to GGSYSAAGAVSG (referred as +3), using the QuickChange site-directed mutagenesis kit (Stratagene). All mutations were confirmed by sequencing. Chinese hamster ovary (CHO-K1) cells were used to express WT and mutant BK channels as described previously [2, 23].

\section{Electrophysiology}

All recordings were performed at room temperature $\left(22-25^{\circ} \mathrm{C}\right)$ in the inside-out configuration of patch-clamp technique using an Axon 200-B amplifier. Patch pipettes were fabricated from disposable micro-pipettes (Drummond Scientific) in two stages on a PP-83 puller (Narishige Scientific Instrument Lab, Tokyo) to a tip diameter of 0.5-1.0 $\mu \mathrm{m}$ and fire-polished on a MG-83 microforge (Narishige Scientific Instrument Lab, Tokyo) to give an average resistance of 5-10 M $\Omega$ in recording solution. Negative pressure was applied via the patch pipette using a pneumatic transducer tester (DPM-IB, BIO-TEK Instruments INC.VT.). Currents were sampled at $5 \mathrm{kHz}$ and filtered at $1.5-2.9 \mathrm{kHz}$ via a four-pole low pass Bessel filter. The program package Clampfit 8.0 was used for data acquisition and analysis. Continuous recordings of 2000-4 $000 \mathrm{~ms}$ were used to estimate the channel open probability $\left(P_{\mathrm{o}}\right)$. The pipette solution contained $145 \mathrm{mM}$ potassium-gluconate, $1 \mathrm{mM}$ EGTA, $10 \mathrm{mM}$ HEPES, and $5 \mathrm{mM}$ glucose with $\mathrm{pH}$ adjusted to 7.4. To minimize potential $\mathrm{Ca}^{2+}$-dependent $\mathrm{SAKCaC}$ activation, EGTA was used to eliminate possible cytoplasmic $\mathrm{Ca}^{2+}$ concentration via putative $\mathrm{Ca}^{2+}$-permeable stretch-activated channels and/or stretch-induced $\mathrm{Ca}^{2+}$ leakage. The bath solution contained the same components as the pipette solution, except for inclusion of indicated $\mathrm{Ca}^{2+}$ concentrations. For $\mathrm{Ca}^{2+}$ concentration lower than $1 \mu \mathrm{M}$, proper concentrations of $\mathrm{CaCl}_{2}$ and $1 \mathrm{mM}$ EGTA were used, on the basis of calculation using the EQCAL program (Biosoft, MO). All reagents were purchased from Sigma. 


\section{Acknowledgments}

We thank Ms Mekie Takahashi, Ms Ritsuko Kanda (Nagaya University, Japan), Dr Changliang Fu and Dr Shouqing Lu (Institute of Mechanics, Chinese Academy of Sciences) for technical assistance. This work was partly supported by research grants from the National Natural Science Foundation of China (10602031) and Grants-in-aid for Scientific Research on Priority Areas (\#15086270 to M.S.) and Creative Research (\#16GS0308 to M.S.) from the Ministry of Education Science Sports and Culture, Japan.

\section{References}

1 Gustin MC, Zhou XL, Martinac B, Kung C. A mechanosensitive ion channel in the yeast plasma membrane. Science 1988; 242:762-765.

2 Qi Z, Chi SP, Su XX, Naruse KJ, Sokabe M. Activation a mechanosensitive BK channel by membrane stress created with amphipaths. Mol Membr Biol 2005; 22:519-527.

3 Kloda A, Lua L, Hall R, Adams DJ, Martinac B. Liposome reconstitution and modulation of recombinant N-methyl-D-aspartate receptor channels by membrane stretch. Proc Natl Acad Sci USA 2007; 104:1540-1545.

4 Hamill OP, Martinac B. Molecular basis of mechanotransduction in living cell. Physiol Rev 2001; 81:685-740.

5 Bahn YS, Xue CY, Idnurm A, Rutherford JC, Heitman J, Cardenas MF. Sensing the environment: lessons from fungi. Nat Rev Microbiol 2007; 5:57-69.

6 Moser T, Brandt A, Lysakowski A. Hair cell ribbon synapses. Cell Tissue Res 2006; 326:347-359.

7 Christensen O. Mediation of cell volume regulation by $\mathrm{Ca}^{2+}$ influx through stretch-activated channels. Nature 1987; 330:66-68.

8 Welsh MJ, Price MP, Xie J. Biochemical basis of touch perception: mechanosensory function of degenerin/epithelial $\mathrm{Na}^{+}$ channels. J Biol Chem 2002; 277:2369-2372.

9 Gasull X, Ferrer E, Llobet A, et al. Cell membrane stretch modulates the high-conductance $\mathrm{Ca}(2+)$-activated $\mathrm{K}^{+}$channel in bovine trabecular meshwork cells. Invest Ophthalmol Vis Sci 2003 ; 44:706-714.

10 Lee J, Ishihara A, Oxford G, Johson B, Jacobson K. Regulation of cell movement is mediated by stretch-activated calcium channels. Nature 1999; 400:382-386.

11 Saint DA. Stretch-activated channels in the heart: their role in arrhythmias and potential as antiarrhythmic drug targets. Drug Dev Res 2002; 55:53-58.

12 Knaus HG, Schwarzer C, Koch RO, et al. Distribution of highconductance $\mathrm{Ca}^{2+}$-activated $\mathrm{K}^{+}$channels in rat brain: targeting to axons and nerve terminals. J Neurosci 1996; 16:955-963.

13 McCartney CE, McClafferty H, Huibant JM, Rowan EG, Shipston MJ, Rowe CM. A cysteine-rich motif confers hypoxia sensitivity to mammalian large conductance voltage and $\mathrm{Ca}$ activated K (BK) channel a-subunits. Proc Natl Acad Sci USA 2005; 102:17870-17875.

14 Loane DJ, Lima PA, Marrion NV. Co-assembly of N-type $\mathrm{Ca}^{2+}$ and BK channels underlies functional coupling in rat brain. $J$ Cell Sci 2007; 120:985-995.

15 Qian X, Niu XW, Magleby KL. Intra-and intersubunit coopera- tivity in activation of BK channels by $\mathrm{Ca}^{2+} . J$ Gen Physiol 2006; 128:389-404.

16 Gribkoff VK, Starrent JE, Dworetzky SL. Maxi-K potassium channels: form, function, and modulation of a class of endogenous regulators of intracellular calcium. Neuroscientist 2001; 7:166-177.

17 Mallouk N, Allard B. Stretch-induced activation of $\mathrm{Ca}^{2+}$-activated $\mathrm{K}(+)$ channel in mouse skeletal muscle fibers. Am J Physiol Cell Physiol 2000; 278:C473-C479.

18 Pacha J, Frindt G, Sackin H, Palmer LG. Apical maxi K channels in intercalated cells of CCT. Am J Physiol 1991; 261:F696-F705.

19 Dopico AM, Kirber MT, Singer JJ, Walsh JV. Membrane stretch directly activates large conductance $\mathrm{Ca}^{2+}$-activated $\mathrm{K}^{+}$channels in mesenteric artery smooth muscle cells. Am J Hypertens 1994; 7:82-89.

20 Allard B, Couble ML, Magloire H, Bleicher F. Characterization and gene expression of high conductance calcium-activated potassium channel displaying mechanosensitivity in human odontoblasts. J Boil Chem 2000; 275:25556-25561.

21 Mienville JM, Barker JL, Lange DG. Mechanosensitive properties of BK channels from embryonic rat neuroepithelium. $J$ Membr Biol 1996; 153:211-216.

22 Kawakubo T, Naruse KJ, Matsubara T, Hotta N, Sokabe M. Characterization for a newly found stretch-activated $\mathrm{K}_{\mathrm{Ca}, \mathrm{ATP}}$ channel in cultured chick ventricular myocytes. Am J Physiol Heart Circ Physiol 1999; 276:H1827-H1838.

23 Tang QY, Qi Z, Naruse K, Sokabe M. Characterization of a functionally expressed stretch-activated BKca channel cloned from chick ventricular myocytes. J Membr Biol 2003; 196:183-200.

24 Naruse KJ, Tang QY, Sokebe M. STREX exon makes $\mathrm{Ca}^{2+}$. activated big $\mathrm{K}^{+}$channels mechanosensitive. Biophys $J$ 2004; 86:545A.

25 Jiang YX, Pico A, Cadene M, Chait BT, Mackinnnon R. Structure of the RCK domain from the E. coli $\mathrm{K}^{+}$channel and demonstration of its presence in the human BK channel. Neuron 2001; 29:593-601.

26 Jiang YX, Lee A, Chen JY, Cadene M, Chait BT, Mackinnon R. Crystal structure and mechanism of a calcium-gated potassium channel. Nature 2002; 417:515-522.

27 Niu XW, Qian X, Magleby KL. Linker-gating ring complex as passive spring and $\mathrm{Ca}^{2+}$-dependent machine for a voltage and $\mathrm{Ca}^{2+}$-activated potassium channel. Neuron $2004 ; \mathbf{4 2 : 7 4 5 - 7 5 6 .}$

28 Stumpff F, Strauss O, Boxberger M, Wiederholt M. Characterization of maxi-K-channel in bovine trabecular meshwork and their activation by cyclic guanosine monophosphate. Invest Ophthalmol Vis Sci 1997; 38:1883-1892.

29 Liu XJ, Chang YC, Reinhart PH, Sontheimer H. Cloning and characterization of glioma $\mathrm{BK}$, a novel $\mathrm{BK}$ channel isoform highly expressed in human glioma cells. J Neurosci 2002; 22:1840-1849.

30 Lingle CJ. Gating rings formed by RCK domains: keys to gate opening. J Gen Physiol 2007; 129:101-107.

31 Salkoff L, Butler A, Ferreira G, Santi C, Wei A. High-conductance potassium channels of the SLO family. Nat Rev Neurosci 2006; 7:921-931.

32 Mallouk N, Jacquemond V, Allard B. Elevated subsarcolemmal $\mathrm{Ca}^{2+}$ in mdx mouse skeletal muscle fibers detected with $\mathrm{Ca}^{2+}$ activated $\mathrm{K}^{+}$channels. Proc Natl Acad Sci USA 2000; 97:49504955 . 
33 Yuan CB, O`Connell RJ, Jacob RF, Mason RP, Treistman SN. Regulation of the gating of BKca channel by lipid bilayer thick- ness. J Biol Chem 2007; 282:7276-7286.

(Supplementary information is linked to the online version of the paper on the Cell Research website.) 